\title{
Successful and Complete Recovery of the Ulnar Nerve After Eight Years of Chronic Injury Through Local Steroid Injections: A Case Report
}

\author{
Diab A. Bani Hani - Khaled Z. Alawneh - Abdelwahab J. Aleshawi · \\ Akram I. Ahmad - Liqaa A. Raffee - Ala"a A. Alhowary · \\ Majdi AlQawasmeh · Bashar Abuzayed
}

Received: October 30, 2019 / Published online: January 3, 2020

(C) The Author(s) 2020

\section{ABSTRACT}

Peripheral neuropathy is a common neurodegenerative disease, with vastness of inducers and causalities. The acquired form peripheral neuropathy can be caused by traumatic injuries caused by nerve lacerations or compressions. Such injuries are usually followed by Wallerian degeneration, and inflammatory reaction. We present a case of a 33-year-old female with a chronic loss of the ulnar nerve function for 8 years after traumatic laceration. After that, she regained the functions of ulnar nerve after nerve stimulation by peri-ulnar nerve injection of methylprednisolone and lidocaine. The theory behind using steroids is related to the fact

Enhanced Digital Features To view enhanced digital features for this article go to https://doi.org/10.6084/ m9.figshare.11379465.

D. A. Bani Hani $(\bowtie) \cdot$ A. A. Alhowary

Department of Anesthesia and Pain Medicine, Faculty of Medicine, Jordan University of Science and Technology, Irbid, Jordan

e-mail: dabanihani@just.edu.jo

\section{K. Z. Alawneh}

Department of Diagnostic Radiology and Nuclear Medicine, Faculty of Medicine, Jordan University of Science and Technology, Irbid, Jordan

A. J. Aleshawi · A. I. Ahmad Faculty of Medicine, Jordan University of Science and Technology, Irbid, Jordan that the immune system could induce a secondary injury that interferes with the recovery. Many studies have shown effectiveness in using steroids alone or when combined with other substances on nerve regeneration in animal models. We believe that this is the first report of nerve recovery using local steroidal injections after a traumatic injury.

Keywords: Complete recovery; Local injection; Methylprednisolone; Peripheral neuropathy; Ulnar nerve 


\section{Key Summary Points}

Why carry out this study?

Many studies showed positive effects of steroid alone or when combined with other materials on nerve regeneration in animals.

One report showed a recovery of neglected neuropathy in a leprosy patient using steroids.

The aim of this article is to describe a successful and complete recovery of the ulnar nerve in a 33-year-old female who sustained a traumatic laceration injury.

\section{What was learned from the study?}

We believe that this is the first report of nerve recovery using local steroid injection after trauma that lead to chronic nerve dysfunction.

\section{INTRODUCTION}

Peripheral neuropathy is one of the most common neurodegenerative diseases with a prevalence of $2.4-8 \%$ [1]. Such diseases can affect multiple neural functionalities in several peripheral nerves, or one nerve initiated by different etiologies. Neuropathic functionality symptoms can be divided into sensory and motor. Sensory symptoms include pain, burning sensation, tingling sensation, numbness, tightness, and sensory ataxia [2]. On the other hand, motor symptoms include muscle cramps, stiffness, weakness, and wasting [2]. Neuropathic pain is prevalent, presenting in approximately two-thirds of patients with peripheral neuropathy and varies with the etiological cause; for example, malignancy-related neuropathic pain would be present in $41.3 \%$ of patients with cancer [2,3]. The disability or non-functionality caused by peripheral neuropathy was found to correlate with a decrease in quality of life, and it has been demonstrated that painful peripheral neuropathy is associated with less quality of life [3].

The acquired form of the disease can be caused by physical injury, systemic or metabolic disorders (as diabetes), vitamin B-12 deficiency, chronic kidney disease, malignancy, infections (as leprosy), alcoholism, autoimmune disorders, exposure to toxic compounds, and ageing [4-12]. On the other hand, the inherited form of peripheral neuropathy is a group of disorders commonly known as Charcot-Marie-Tooth disease $[2,13]$.

Also, depending on the affecting nerves, peripheral neuropathy has three sub-classifications; mononeuropathy, which is a result of an entrapped or traumatized nerve or mononeuropathy multiplex, which is linked to chronic diseases like leprosy, and polyneuropathy, which is a part of systemic, metabolic, or toxic disorder [14]. The most common cause for mononeuropathy is a peripheral nerve physical injury. The most common form of this physical injury is the traumatic form including nerve lacerations (trans-sections) and compression (crush) [15]. Trauma will induce tissue destruction in which distal nerve stumps undergo cellular changes known as Wallerian degeneration, this process is not observed directly after physical injury [16]. A secondary immune-mediated injury is then noticed, which subsequently inhibits the repair and regeneration of peripheral axons and myelin sheaths [17]. During the first week after nerve injury, many proinflammatory mediators trigger tissue destruction, increase Schwann cell numbers, activate resident nonneuronal cells to produce more quantities of inflammatory mediators, and recruit leukocytes to degenerated nerves [18]. This feedback loop of nerve destruction, cell proliferation, immune cell, and proinflammatory cytokine release makes the nerve inflamed for long periods after trauma [18]. In the days following nerve trauma (within the chronic phase), the distal stump may undergo structural changes [18]. There is no doubt that the inflammatory reactions enhance this structural process. In this article, the "peripheral neuropathy" indicates the mononeuropathy that would result from traumatic causes. 
Several animal studies have investigated the role of anti-inflammatory drugs such as steroids in the stimulating of atrophied nerves [19]. The correlation between immunosuppression properties and the postulated neurotrophic effects of steroids can have an outstanding therapeutic benefit for peripheral nerve injuries [19]. In 1974, Varrassi et al. first described the effect of corticosteroid in the restoration of blood circulation [20].

The aim of this article is to describe a successful and complete recovery of an ulnar nerve traumatic laceration injury in a 33-year-old female after 8 years of chronic injury using methylprednisolone injections.

\section{Consent for Publication and Ethical Approval}

Written informed consent was obtained from the patient for publication of this case report and any accompanying images. Institutional review board approval is not required for this case report.

\section{CASE REPORT}

A 33-year-old married female with a non-significant past medical history presented in 2008 to a primary emergency hospital complaining of right wrist laceration which developed after a traumatic injury caused by a falling glass. At the hospital, the patient was sent to the theater and exploration was performed. Injuries to the ulnar artery, ulnar nerve, flexor carpi ulnaris muscle, and flexor digitorum superficialis muscle were detected. The surgeon at that hospital was only skilled in the correction and repair of arterial injuries.

The patient was then referred to our hospital in order to correct the muscle tendons and nerve injuries. A second operation to repair the muscular tendons was performed. During the operation, partial laceration of the ulnar nerve with an extensive neuroma was observed. The lesion was near the wrist joint and no entrapment was found. Accordingly, she was referred for another specialized surgeon in an attempt to remove the neuroma with a possible nerve grafting.
Table 1 Nerve conduction studies in 2008

\begin{tabular}{lll}
\hline Nerve site & Latency & Amplitude \\
\hline Motor function & & \\
Right ulnar nerve-ADM (wrist) & $5.3 \mathrm{~ms}$ & $4.0 \mathrm{mV}$ \\
Right ulnar nerve-FDI (wrist) & $3.3 \mathrm{~ms}$ & $1.8 \mathrm{mV}$ \\
Sensory function & & \\
Mid-palm (ulnar) & $\mathrm{NR} \mathrm{ms}$ & $\mathrm{NR} \mu \mathrm{V}$ \\
\hline
\end{tabular}

$N R$ not responding

Unfortunately, the third surgery was delayed for more than 5 months due to the patient's financial and insurance issues. At that time, the surgeon believed that no surgical approach would be effective. Subsequently, nerve conduction studies were performed, which revealed severe acute and chronic axonal injury to the right ulnar nerve with more involvement in the branch that supplies the first dorsal interosseous muscle compared to the branch that supplies the abductor digiti minimi muscle. Moreover, a complete loss of sensory function of the ulnar nerve was observed (Table 1). As a result, the patient was referred for physio- and occupational-therapy sessions.

In 2015 , the patient presented to our outpatient clinic with the aforementioned history for the possible nerve stimulation. On examination, the patient's right hand was in an ulnar claw position. A complete loss of sensation in the ulnar nerve distribution was monitored. No additional nerve conduction studies were required.

Based on our suggested theory about the correlation between immunosuppression and peripheral nerve injuries, the decision was to try the nerve stimulation using peri-ulnar nerve

Table 2 Nerve conduction study in 2016

\begin{tabular}{lll}
\hline Nerve site & Latency & Amplitude \\
\hline Motor function & & \\
Right ulnar nerve-ADM (wrist) & $3.4 \mathrm{~ms}$ & $10.8 \mathrm{mV}$ \\
Sensory function & & \\
Right ulnar palm (wrist) & $1.6 \mathrm{~ms}$ & $17 \mu \mathrm{V}$ \\
\hline
\end{tabular}


steroid injection of $40 \mathrm{mg}$ methylprednisolone and $1 \mathrm{cc}$ of $1 \%$ lidocaine (this treatment regimen was applied on three sessions with an interval of 4 weeks). Gradually, the patient started to regain her sensory and motor abilities. In October of 2016, 1 month after the last steroidal injection, another nerve conduction study was performed which revealed normal ulnar nerve function with no evidence of remaining disability in the nerve function (Table 2).

The last follow-up visit was in 2019, where she reported normal function and the examination revealed normal motor and sensory functions.

\section{DISCUSSION}

To the best of our knowledge, this is the first report of successful and complete recovery of traumatic chronic nerve injury by local perineural corticosteroid injections without nerve graft repair or stump re-approximation. The nerve stimulation was achieved after 8 years of suffering from complete sensory and motor loss of the right ulnar nerve. Nashed et al. reported the first case of successful nerve regeneration in humans after neglected neuropathy secondary to leprosy by a local injection of corticosteroids [21]. In addition, several experimental studies demonstrated the therapeutic effect of corticosteroids in the treatment of nerve injury [19]. Feng and Yuan demonstrated that dexamethasone can promote peripheral nerve repair in a rat model of sciatic nerve injury [16]. Furthermore, Ozturk et al. concluded that the synergistic effect of methylprednisolone and ozone can have a beneficial effect regarding nerve regeneration after crush-type nerve injury [22].

Until now, there have been no proven therapeutic agents that can induce axonal regeneration resulting in an improvement in the sensory and motor functionalities after nerve trauma. Following a traumatic injury, axons break down, neural Schwann cells refuse the myelin sheath, and a variety of peripheral immune cells, including mast cells, macrophages, and lymphocytes, are recruited to sites of injury [16]. Immune activation that follows a peripheral nerve injury including the release of inflammatory cytokines along with the recruitment of immune cells plays an important role in degeneration and regeneration of the damaged area [19].

The use of corticosteroids can inhibit the immune reactivity in the site of traumatic injury, resulting in a pressure decrease on the neuronal axons and augments nerve regeneration and re-myelination [19]. In the case of our patient, an outstanding recovery of both sensory and motor abilities was observed. The recovery was induced by peri-ulnar nerve steroid injection of methylprednisolone. There were no reported complications or side effects from this therapy. The advantage of local corticosteroid therapy is to deliver the medication at the site of action in higher concentrations compared to the systemic route with minimal systemic side effects.

Neuroactive steroidal substances have shown a promising neuroprotective effect in different experimental animal models $[16,19,22]$. In an experimental study conducted by Azcoitia et al., neuroactive steroids appeared to influence many physiological events involved in neural regeneration of rats' peripheral nerves, such as synthesis of myelin proteins, Schwann cell proliferation, and function of the axonal compartments through multiple signaling mechanisms [23]. In addition, progesterone and pregnenolone induced a neuronal regeneration when applied locally to the site of peripheral neuropathy, which can be explained by the ability of such neuroactive steroids to counteract the decrease in myelin in transected sciatic nerve of mice [24]. Melcangi et al. have illustrated treatment with neuroactive steroids and were also able to modulate the expression of myelin proteins, such as glycoprotein zero in an experimental model of sciatic nerve transection $[19,25]$.

Furthermore, similar interesting results have been obtained in the same experimental model in mice, using $17 \beta$-estradiol as a neuroactive steroid [26]. In Feng and Yuan's model, they demonstrated that dexamethasone promotes peripheral nerve repair in rats through the inhibition of CD3-positive cell infiltration as well as the upregulation of GAP-43 expression 
[16]. Glucocorticoids were reported to be able to stimulate the transcription of glycoprotein and peripheral myelin protein 22 promoters in Schwann cells [27]. Within the central nervous system, microglia cells are present in a physiological branched form. When stimulated, they become proinflammatory. In elderly people, microglia are present in a primed form, which is responsible for a more intense and long-term inflammation. This abnormally strong reaction may be the main cause for persistent neuroinflammation and irreversible neuronal damage. The primed microglia present at the spinal cord level and in the thalamus seems to facilitate the initiation of chronic and neuropathic pain. Furthermore, proinflammatory cytokines overreleased by activated microglia may also damage the integrity of the white substance and the ultrastructure of the myelin sheath [28].

Regarding their anti-inflammatory effect, local anesthetics have been shown to affect neutrophils directly, as well as macrophage and monocytes. Ropivacaine and lidocaine decreased tumor necrosis factor alpha- $\alpha$-induced upregulation of CD11b/CD18 surface expression on neutrophils. Thus, local anesthetics decrease neutrophils adherence, migration, and accumulation at the site of inflammation [29]. Their effect would attribute in part to the observation in this study.

\section{CONCLUSIONS}

Injury to peripheral nerves results in temporary or life-long neuronal dysfunction that can subsequently lead to economic or social disabilities. The utilization of local steroids and local anesthetics to stimulate peripheral nerve injury should be investigated through clinical trials and further studies should be conducted. We yet report the first case of ulnar nerve recovery after 8 years of complete function loss through local steroid injections.

\section{ACKNOWLEDGEMENTS}

We thank the participant patient of the study.
Funding. No funding or sponsorship was received for this study or publication of this article.

Authorship. All named authors meet the International Committee of Medical Journal Editors (ICMJE) criteria for authorship for this article, take responsibility for the integrity of the work as a whole, and have given their approval for this version to be published.

Disclosures. There are no grants, sponsors, funding sources, or direct financial support to disclose. Diab A. Bani Hani, Khaled Z. Alawneh, Abdelwahab J. Aleshawi, Akram I. Ahmad, Liqaa A. Raffee, Ala"a A. Alhowary, Majdi AlQawasmeh and Bashar Abuzayed have nothing to disclose.

Compliance with Ethics Guidelines. Written informed consent was obtained from the patient for publication of this case report and any accompanying images. Institutional review board approval is not required for a case report.

Data Availability. Data sharing is not applicable to this article as no datasets were generated or analyzed during the current study.

Open Access. This article is distributed under the terms of the Creative Commons Attribution-NonCommercial 4.0 International License (http://creativecommons.org/licenses/ by-nc/4.0/), which permits any noncommercial use, distribution, and reproduction in any medium, provided you give appropriate credit to the original author(s) and the source, provide a link to the Creative Commons license, and indicate if changes were made.

\section{REFERENCES}

1. Hanewinckel R, Drenthen J, van Oijen M, Hofman A, van Doorn PA, Ikram MA. Prevalence of polyneuropathy in the general middle-aged and elderly population. Neurology. 2016;87(18):1892-8.

2. Girach A, Julian TH, Varrassi G, Paladini A, Vadalouka A, Zis P. Quality of life in painful peripheral 
neuropathies: a systematic review. Pain Res Manag. 2019;2019:2091960.

3. Zis P, Paladini A, Piroli A, McHugh PC, Varrassi G, Hadjivassiliou M. Pain as a first manifestation of paraneoplastic neuropathies: a systematic review and meta-analysis. Pain Ther. 2017;6:143-51.

4. Prior R, van Helleputte L, Benoy V, Van Den Bosch L. Defective axonal transport: a common pathological mechanism in inherited and acquired peripheral neuropathies. Neurobiol Dis. 2017;105: 300-20.

5. Feldman EL, Nave KA, Jensen TS, Bennett DLH. New horizons in diabetic neuropathy: mechanisms, bioenergetics, and pain. Neuron. 2017;93(6): 1296-313.

6. Papanas N, Ziegler D. Risk factors and comorbidities in diabetic neuropathy: an update 2015. Rev Diabet Stud. 2015;12(1-2):48-62.

7. Gibbons CH. Treatment-induced neuropathy of diabetes. Curr Diab Rep. 2017;17(12):127.

8. Chopra K, Tiwari V. Alcoholic neuropathy: possible mechanisms and future treatment possibilities. Br J Clin Pharmacol. 2012;73(3):348-62.

9. Staff NP, Grisold A, Grisold W, Windebank AJ. Chemotherapy-induced peripheral neuropathy: a current review. Ann Neurol. 2017;81(6):772-81.

10. Geber C, Breimhorst M, Burbach B, et al. Pain in chemotherapy-induced neuropathy-more than neuropathic? Pain. 2013;154(12):2877-87.

11. Little AA, Albers JW. Clinical description of toxic neuropathies. Handb Clin Neurol. 2015;131: 253-96.

12. Kumar N. Neurologic aspects of cobalamin (B12) deficiency. Handb Clin Neurol. 2014;120:915-26.

13. Diouf B, Crews KR, Lew G, et al. Association of an inherited genetic variant with vincristine-related peripheral neuropathy in children with acute lymphoblastic leukemia. JAMA. 2015;313(8):815-23.

14. Misra UK, Kalita J, Nair PP. Diagnostic approach to peripheral neuropathy. Ann Indian Acad Neurol. 2008;11:89.

15. Burnett MG, Zager EL. Pathophysiology of peripheral nerve injury: a brief review. Neurosurg Focus. 2004;16(5):E1.

16. Feng X, Yuan W. Dexamethasone enhanced functional recovery after sciatic nerve crush injury in rats. Biomed Res Int. 2015;2015:627923.
17. Zochodne DW. The microenvironment of injured and regenerating peripheral nerves. Muscle Nerve Suppl. 2000;9:S33-8.

18. Mietto BS, Mostacada K, Martinez AM. Neurotrauma and inflammation: CNS and PNS responses. Mediators Inflamm. 2015;2015:251204.

19. Melcangi RC, Garcia-Segura LM. Therapeutic approaches to peripheral neuropathy based on neuroactive steroids. Expert Rev Neurother. 2006;6(8):1121-5.

20. Varrassi G, Marino B, Cosmi EV. Influence of methylprednisolone sodium succinate on the microcirculation. Acta Anaesth It. 1974;25:159-71.

21. Nashed SG, Rageh TA, Attallah-Wasif ES, Abd-Elsayed AA. Intraneural injection of corticosteroids to treat nerve damage in leprosy: a case report and review of literature. J Med Case Rep. 2008;2:381.

22. Ozturk O, Tezcan AH, Adali Y, et al. Effect of ozone and methylprednisolone treatment following crush type sciatic nerve injury. Acta Cir Bras. 2016;31(11): 730-5.

23. Azcoitia I, Leonelli E, Magnaghi V, Veiga S, GarciaSegura LM, Melcangi RC. Progesterone and its derivatives dihydroprogesterone and tetrahydroprogesterone reduce myelin fiber morphological abnormalities and myelin fiber loss in the sciatic nerve of aged rats. Neurobiol Aging. 2003;24(6): 853-60.

24. Koenig HL, Schumacher M, Ferzaz B, et al. Progesterone synthesis and myelin formation by Schwann cells. Science. 1995;268(1-3):1500-3.

25. Melcangi RC, Azcoitia I, Ballabio M, et al. Neuroactive steroids influence peripheral myelination: a promising opportunity for preventing or treating age-dependent dysfunctions of peripheral nerves. Prog Neurobiol. 2003;71(1):57-66.

26. Islamov RR, Hendricks WA, Jones RJ, Lyall GJ, Spanier NS, Murashov AK. 17Beta-estradiol stimulates regeneration of sciatic nerve in female mice. Brain Res. 2002;943(2):283-6.

27. Schumacher M, Guennoun R, Mercier G, et al. Progesterone synthesis and myelin formation in peripheral nerves. Brain Res Brain Res Rev. 2001;37(1-3):343-59.

28. Varrassi G, Fusco M, Coaccioli S, Paladini A. Chronic pain and neurodegenerative processes in elderly people. Pain Pract. 2015;15:1-3.

29. Cruz FF, Rocco PR, Pelosi P. Anti-inflammatory properties of anesthetic agents. Crit Care. 2017;21: 67. 